\title{
Schutzumschläge und Qualitätsbezeugungen
}

\author{
Überlegungen zur Peinlichkeit des Populären
}

Ingrid Tomkowiak

Haben Sie eigentlich Harry PotTer gelesen? Ja, schon, aber in der Ausgabe für Erwachsene, die mit dem dezenteren Schutzumschlag. Und ausserdem muss ich wissen, was mein Sohn so verschlingt. - Lesen Sie Fifty Shades of Grey? Ja, aber nur auf dem E-Reader, da sieht dann keiner, was ich lese. - Schauen Sie Serien? Nur die wirklich guten, also Quality TV, da hat sich ja viel getan in den letzten Jahren, und ich will doch auch mitreden können. - Und die Verfilmungen der Romane von Rosamunde Pilcher, sehen Sie die? Nein, so etwas schaue ich mir gar nicht erst an, das wäre ja peinlich! - Und was halten Sie von den alten Science Fiction-Filmen eines Ed Wood oder von Raumpatrouille oder den Edgar Wallace-Filmen? Na, das ist etwas anderes: Die sind so peinlich, dass sie schon wieder gut sind!

Dieses kleine Interview ist fiktiv, doch könnte es - oder ein Teil daraus - heute genau so stattfinden. Als Alltagsdialog ist das schon weniger wahrscheinlich, denn solche Fragen stellt man ja niemandem, man will ja niemanden in eine peinliche Situation bringen! Oft genug werde ich im Zug erstaunt beäugt, wenn ich Kinderbücher lese oder die neueste Biographie über Johnny Depp. Es kommt mir die Redewendung: »Sage mir, was du liest, und ich sage dir, was du bist! «, in den Sinn. Oder: "Nur Dumme lesen Schund!«, wie ein Aushang in einer deutschen Buchhandlung etwa 1910 behauptete. ${ }^{1}$ Und da möchte man doch weiss Gott nicht dazu gehören! Und so wird Donna Leons neuester Brunetti-Fall auch ganz bestimmt nicht für die eigene Lektüre, sondern für die der sso etwas liebenden Tante gekauft.

Ich habe es allerdings leicht und kann mich immer mit meinem Beruf rausreden, andere berufen sich auf ihre Kinder oder die immerhin doch vorhandene Qualität dessen, was sie konsumieren, oder sie verstecken ihre Lektüre hinter einem Diskretion garantierenden Schutzumschlag. Wie gut, dass man auf manchen Computerbild-

\footnotetext{
1 Vgl. Kaspar Maase, Die Kinder der Massenkultur. Kontroversen um Schmutz und Schund seit dem Kaiserreich, Frankfurt a.M. 2012, 244: Die Plakataufschrift lautete: "Nur Dumme lesen solche Schundromane!" Der vorliegende Beitrag hat dieser Untersuchung und anderen Schriften von Kaspar Maase wesentliche Informationen zu verdanken.
} 
schirmen oder Tablets nichts erkennen kann, wenn man von der Seite darauf sieht! Und wenn zwar vielleicht die Konsumierenden kein Problem damit haben, dass andere sehen, wie sie sich im Zug Katastrophenfilme reinziehen, so gibt es ganz sicher diejenigen, die darüber die Nase rümpfen und sich `fremdschämen`:Wie kann man nur!

Populäres kann also peinlich sein, und die Rezeption von Populärem bzw. das Vergnügen daran bedarf offenbar besonderen Schutzes und besonderer Rechtfertigung. Auch in Zeiten wie diesen, in denen es in der Literatur von populärkulturellen Zitaten nur so wimmelt und Intellektuelle sich mit der produktiven Rezeption des >Illegitimen nicht nur schmücken, sondern profilieren ${ }^{2}$, ist es vielen Menschen unangenehm, wenn sie bei der Lektüre eines Bestsellers rerwischt werden oder in der Kinopause meinen erklären zu müssen, warum sie nun doch in den letzten Teil des Hobbit gegangen sind.

Hinter solchen Erklärungen oder Ausreden stehen tiefgreifende Prozesse kultureller Delegitimierung, Disziplinierung und Selbstzivilisierung. Vielleicht werden Erwachsene, die als Kind die heissgeliebten Comics nicht von ihrem Taschengeld bezahlen durften und als Jugendliche die Bravo nur heimlich lesen konnten, das Gefühl des Verbotenen nie ganz los.

\section{Kulturelle Einprägungen}

Die Geschichte der Wahrnehmung des Populären bis in die Gegenwart hinein ist nicht zu verstehen ohne die Geschichte der Widerstände und Kämpfe dagegen. Damit verbundene Wahrnehmungs-, Deutungs- und Handlungsmuster aus dem 18. und 19. Jahrhundert reproduzieren sich zum Teil bis heute.

"Aber vorsichtig, lass dich von dem, was du liest, nicht vergiften."

Tobte im 18. Jahrhundert die Paola in Umberto Eco, Die "Lesesucht«-Debatte gegen das geheimnisvolle Flamme der Königin Roman-Lesen der Frauen, sorgte die "Zweite Leserevolution" ab ca. 1850 mit ihrer gesteigerten Lesestoff-Produktion für ein breiteres lesefä-

2 Vgl. Thomas Becker, Einleitung, in: Ästhetische Erfahrung der Intermedialität. Zum Transfer künstlerischer Avantgarden und sillegitimer Kunst im Zeitalter von Massenkommunikation und Internet, hg. v. dems., Bielefeld 2011, 7-31, hier 11-14: In Anlehnung an Umberto Ecos Studie Apokalyptiker und Integrierte (s.u.) spricht Becker in solchem Zusammenhang von den Hyperintegrierten bzw. einem postmodernisierten Adornismus. 
higes Publikum neben der ohnehin praktizierten politischen und moralischen Zensur für eine Kontrolle, Lenkung und Beschränkung der unterhaltenden Lektüre durch Schule, Kirche und Volkslesevereine. Dabei ging es denen auch um die Verteidigung der kulturellen Autorität der Bildungsschichten. Produzenten und Vertreiber kommerzieller populärer Lesestoffe dagegen wurden als Gegner behandelt; ihr unternehmerisches Interesse am Massengeschäft mit dem Lesebedürfnis der Unterschichten galt als ebenso unmoralisch wie ihre Produkte. Ihre Ablehnung basierte auf der Vorstellung von Unterhaltung als Gefahr - Selbstgefährdung und Gefährdung der Ordnung durch Sittenlosigkeit und Leichtsinn, Verachtung gesetzlicher, moralischer und religiöser Normen. Lesen hatte dem Primat der Nützlichkeit zu folgen; Lektüre als Selbstzweck, Vergnügen oder Stimulus der Einbildungskraft war massiven Abwehrprozessen unterworfen.

Die auf Distinktion bedachte Normsetzungsmacht der Bildungsschichten sorgte nicht nur für die Delegitimation populärer Lesestoffe, sondern auch für die kulturelle und soziale Abwertung ihrer Leser. Der über Jahrzehnte des 20. Jahrhunderts

Unter dem Kissen lag nicht mehr das Buch, das sonst allnächtlich nach verbotenem Brauch mit letzter Kraft dort hingeschoben wurde. [...] Die Krankheit hatte kommen müssen, um mir ein reinliches Gewissen zu verschaffen. Walter Benjamin, Berliner Kindheit um 1900, 38 währende und gesetzlich gestützte Kampf gegen den sogenannten Schund und Schmutz stand nicht nur, aber insbesondere zu Beginn unter dem Postulat, Populärkultur sei ein Unterschicht- und Jugendproblem, sei minderwertig und sozial gefährlich, denn ihre Nutzer seien ästhetisch primitiv und potenziell

Roberts Antlitz verfinsterte sich. "Woher hast du diesen Schund?« Fredy zeigte auf mich. Ich färbte mich puterrot, und Robert fasste dies als das Eingeständnis einer Schuld auf. Er zerriss das Heft mittendurch und warf die Fetzen vor mich hin. [...] Da verstummte ich, denn mir wurde bewusst, dass Fredy und ich Verbotenes gelesen hatten.

Paul Wehrli, Martin Wendel. Roman einer Kindheit, $77 \mathrm{f}$. verroht. Weil Liberalität gegenüber profitorientierter Kulturindustrie Gefahren für Jugend, Kultur und Gemeinwesen schaffe, könne nur die Autorität der Bildungsschichten vor den Gefahren bewahren. Um die Volksmassen also zu hygienisieren, bediente man sich einer ausgesprochen symbolischen Politik und einer Metaphorik des Rohen, Schmutzigen und sogar Krankhaften. Die Methoden waren entsprechend drastisch: Massiver Druck wurde auf SchülerInnen und Eltern ausgeübt, 
die öffentliche Meinung aufgestachelt - man könnte von moral panic sprechen -, es kam zu Boykottaufrufen, Durchsuchungen, Sammelund Tauschaktionen und öffentlichen Verbrennungen der verhassten Lesestoffe. Und so gehört das Verstecken von Lesestoff oder verbotenes Lesen im Verborgenen zur Erfahrung von Kindern und Jugendlichen vieler Generationen; heimliche LektüreOrte (wie auch Orte des Lesestofferwerbs, -tauschs oder seiner Ausleihe) sind wichtige Bestandteile ihrer Kindheit und Jugend. Dass es sich bei ihrer Lieblingslektüre um Schund handelte, konnten sie nicht am Text erkennen, sondern am Verbot bzw. der Beschlagnahmung.

Kaspar Maase, der dem Schund-

Herr Brämi hatte bereits mit der Untersuchung begonnen. Er durchstöberte Hefte, Bücher und Tornister, wandte die Buchdeckel um, tastete die Dicke der Hefte ab, griff in die Innentaschen des Ranzens, ob sich vielleicht dort ein Schundheft verborgen hatte. [...] Vierzehn Hefte hat der Lehrer gefunden, das meiste Clemens-Ruby-Hefte, und wir wussten nun, dass auch diese Serie zur Schundliteratur gerechnet wurde. Paul Wehrli, Martin Wendel. Roman einer Kindheit, 83f. schaftlicher Studien gewidmet hat und 2012 die grossangelegte Untersuchung Die Kinder der Massenkultur vorlegte, schätzt die Wirkung der Massnahmen so ein: »Mehrere Jahrzehnte hindurch erfuhren Heranwachsende die Praxen derVolkserzieher schmerzhaft, nicht selten im wörtlichen Sinne hautnah. Die pädagogische Absicht, sie sollten sich für ihre sschlechte Lektüre schämen, erreichte durchaus ihr Ziel; Generationen genossen ihre Lieblingslektüre mit schlechtem Gewissen. $\ll^{3}$

In vielen autobiographisch gefärbten Texten ${ }^{4}$ wird allerdings auch die Ambivalenz der Rezeption populärer Lesestoffe deutlich, ein Schwanken zwischen Faszination und Abwehr. So bewirkte die Aura des Missliebigen und Verbotenen, die die Lektüre im Verborgenen umgab, ganz offenbar eine Intensivierung des Genussmomentes. Gerade weil dieser Lesestoff als minderwertig galt, als Folge der kulturellen Einprägung, erlebte man ihn lustvoll! Würde man sonst all die Risiken eingehen? Ab Beginn der 1950er Jahre zeigte sich seitens des Bildungsbürgertums ein wachsendes Unbehagen gegen-

\footnotetext{
3 Kaspar Maase, Texte und Praxen. Populärliteraturforschung als historische Ethnografie, in: Schweizerisches Archiv für Volkskunde 97 (2001), 43-51, hier 48.

4 Die angeführten Zitate stammen aus:Walter Benjamin, Berliner Kindheit um 1900. Mit einem Nachwort von Theodor W. Adorno. (Fassung letzter Hand und Fragmente früherer Fassungen), Frankfurt a.M. ${ }^{4}$ 2013; Paul Wehrli, Martin Wendel. Roman einer Kindheit, Zürich 1942; Umberto Eco, Die geheimnisvolle Flamme der Königin Loana. Illustrierter Roman, München 2004.
} 
über den Entwicklungen im Buchhandel, darunter dem vermehrten Auftreten von Bestsellern. ${ }^{5}$ Unter dem Titel Wider die Bestsellerseuche brachte Hans Ferdinand Schulz dieses Unbehagen 1952 - kurz nach dem Ende des nationalsozialistischen Regimes - prägnant zum Ausdruck. ${ }^{6}$ Der kurzlebige Bestseller amerikanischen Typs sei eine dem deutschen Wesen nicht entsprechende Entartungserscheinung, von der ein wirklicher Buchhändler sich unbedingt zu distanzieren habe. Wer den Beruf des Buchhändlers in tieferem Sinne erfasst habe, empfinde das Buch als sewigen Wert und dürfe doch nicht im Novitätenbetrieb aufgehen. ${ }^{7}$ Als Frank Thiess acht Jahre später in einer Akademie-Abhandlung über den Bucherfolg nachdachte, beklagte er, dass der Manager-Verleger den Erfolg bestimme - übelster Schriften, deren gierige Lektüre den Realitätssinn zerstöre und zu einem geistigen Analphabetismus führe. Dergleichen könne nur in einem Zeitalter glïcken, in dem die wertbewusste und wertsetzende Bildungsschicht in der Gesellschaft mehr und mehr zerbröckle. ${ }^{8}$

Das Heft brannte auf meinem Leib, und $[\ldots]$ sobald der Lehrer eine Pause eintreten liess, fürchtete ich eine neue Durchsuchung der Bücher, Hefte und Kleider. [...] Ich raste nach Hause, raste wie der Sturmwind einherbraust, hinauf in den dritten Stock, hinein in den Abort, wo ich das Heft in den Luftschacht emporschleuderte. Am Abend las ich es. Es war die schönste Geschichte von Ruby, die mir je vor die Augen gekommen war.

Paul Wehrli, Martin Wendel. Roman einer Kindheit, 86f.
Dieter E. Zimmer, FeuilletonJournalist der ZEIT, führte 1971 eine über mehrere Beiträge laufende Kampagne gegen die "Diktatur der Bestseller" und trat damit eine breite, polemisch geführte Debatte in den Medien los. Wie Schulz, der von "Seuche" gesprochen hatte ${ }^{9}$, und andere, denen "Bestselleritis" der geeignete Ausdruck schien ${ }^{10}$, bediente sich auch Zimmer für seine Vision vom Ruin des Büchermarktes der Metapher der Krankheit. ${ }^{11}$ In dieser kulturkritischen Perpektive drückt sich die Angst vor dem Verlust der

\footnotetext{
5 Vgl. Ingrid Tomkowiak: Schwerpunkte und Perspektiven der Bestsellerforschung, in: Schweizerisches Archiv fürVolkskunde 99 (2003), 49-64.

6 Hans Ferdinand Schulz, Wider die Bestsellerseuche, in: Der Jungbuchhandel 6 (1952), 162-176.

7 Ebd., 162, 167, 175.

8 Frank Thiess, Der Bucherfolg. Ursprung und Wandel, Mainz 1960, $10 \mathrm{f}$.

9 Schulz, Wider die Bestsellerseuche.

${ }^{10}$ Manfred Hellmann, "Es bestsellert sich nicht so." Gedanken und Untersuchungen zu den Bestsellerlisten, in: Bertelsmann Briefe H. 39 (1965), 7-13, hier 13.

${ }^{11}$ Dieter E. Zimmer, Die Diktatur der Bestseller. Steht der Büchermarkt vor dem Ruin?, in: Die ZEIT, 15.10.1971.
} 
ehemals akzeptierten bürgerlichen Werte aus, welche den idealistischen Vorstellungen von Schriftstellern, Verlagswesen und Buchhandel in dieser Zeit noch zugrunde liegen. ${ }^{12}$

Der Kampf gegen vermeintlich schädliche Literatur, so lässt sich zusammenfassend festhalten, ist als Machtkampf in Form des "sozialen Theaters" (Erving Goffman) zu deuten: Im Konflikt zwischen Oben und Unten positionieren sich die Aktionspartner im sozialen Raum. Die Einen setzen die Konstruktion von "Schund" als Mittel symbolischer Gewalt ein und nutzen dabei Praktiken der Selbstermächtigung, Distinktion, Grenzziehung und Stigmatisierung. Diese führen $\mathrm{zu}$ nachhaltigen Einprägungen der "feinen Unterschiede" (Bourdieu):

"Liesest du solchen Schund? Künzli erwiderte nichts und stand wie ein begossener Pudel da. Mir aber fuhr der Schreck durch die Glieder. Das Tun meines ältesten Bruders kam mir in den Sinn, denn der Lehrer zerriss das Heft - ritsch-ratsch - schlug die Fetzen um Künzlis Ohren und sagte scharf: "Weh dir, wenn ich dich noch einmal erwische! « Angewidert warf er die Fetzen in den Papierkorb.

Paul Wehrli, Martin Wendel. Roman einer Kindheit, 79 der snatürlichen Überlegenheit von dem, was die Bildungsschichten unter "Kultur" verstehen, und der 'kulturellen Unwürdigkeit` des Populären und seiner Nutzer. Wer dies verinnerlicht hat, dem/der ist es peinlich, als PopulärkulturNutzerIn entlarvt zu werden.

Doch gibt es auch die Anderen. Sie beugen sich diesem kulturellen Über-Ich nicht, ziehen ihrerseits Grenzen von unten und schaffen sich über »Schund«-Gebrauch Freiräume. Dazwischen anzusiedeln sind wohl die von Thomas Becker so bezeichneten Hyperintegrierten $^{13}:$ Im internalisierten Wissen um die Minderwertigkeit der Populärkultur bzw. das negative Urteil über sie nutzen sie diese nun provokativ.

\section{Neubewertung des Populären?}

Bereits zu Beginn der 1960er Jahre hatte sich Umberto Eco mit der "kritischen Kritik der Massenkultur" auseinandergesetzt, wie es im Untertitel zu seinem Buch Apokalyptiker und Integrierte (1964) heisst. Es bietet eine für den Umgang mit dem Populären in jener Zeit

\footnotetext{
${ }^{12}$ Vgl. Werner Faulstich, Bestandsaufnahme Bestseller-Forschung. Ansätze, Methoden, Erträge, Wiesbaden 1983, 152.

${ }^{13}$ Becker, Einleitung.
} 
wegweisende Reflexion über gegensätzliche Positionen zur "Massenkultur», ergänzt um eigene Überlegungen zu »Kultur-Niveaus", zu Kitsch, zur Ästhetik des Typischen und zur »Struktur des schlechten Geschmacks" sowie um Analysen zum Comic Steve Canyon, zum Mythos von Superman, zur Welt der Peanuts, zu den erzählerischen Strukturen in den James Bond-Romanen von Ian Fleming und zum Verhältnis von Sozialismus und Trost bei Eugène Sue.

In der Einleitung zu diesem Band ${ }^{14}$ stellt Eco unter anderem Vorwürfe der von ihm so bezeichneten "apokalyptischen Tugendhaften" (19) gegen die Massenmedien zusammen - kulturpessimistische Positionen, vor allem im Gefolge der Kritik der Kulturindustrie als Massenbetrug durch Theodor W. Adorno und Max Horkheimer in ihrer Essaysammlung Dialektik der Aufklärung, 1944 erstmals im US-amerikanischen Exil erschienen. Zusammenfassend attestiert Eco den Apokalyptikern eine pessimistische, ja gegnerische Haltung gegenüber der Massenkultur, die diese als Antikultur, als Zeichen unwiderruflichen Zerfalls deute. ${ }^{15}$ Ecos eigene Position geht von den Gegebenheiten aus: "Ob wir es anerkennen oder nicht, das Universum der Massenkommunikation ist unser Universum. $\aleph^{16}$ Man müsse sie deshalb zum Gegenstand der Forschung machen, und diese dürfe sich nicht nur nicht scheuen, »edle Werkzeuge an verpönten Objekten zu gebrauchen", sondern müsse sich auch von dem Vorbehalt verabschieden, dass »ein Erkenntnisinteresse, das sich dem Verderblichen zuwendet, anstössig, gar minderwertig erscheint « ${ }^{17}$.

Im Jahr 1984, als nach zwanzig Jahren (!) die erste deutsche Übersetzung seines Buches erscheint, schreibt Eco eigens ein Vorwort zur deutschen Ausgabe, in dem er unter anderem die selbst gestellte Frage beantwortet, weshalb er sich seit Ende der fünfziger Jahre für die Phänomene der Massenkommunikation interessiere:

"Ich las Comics und Kriminalromane, ich liebte die Filme von Fred Astaire und das Musiktheater des Broadway. Vielleicht schämte ich mich heimlich dafür, da viele berühmte Schriftsteller mir sagten, dass dies alles nichts tauge. [...] Später bewunderte ich Walter Benjamin, weil er, im Unterschied zu seinen Freunden der 'Frankfurter Schules, die Massenkultur mit grosser und wacher Neugier und ohne Vorverurteilung studierte. [...] Heute könnte ich dieselben Themen viel lockerer erörtern, doch damals musste

\footnotetext{
${ }^{14}$ Umberto Eco, Apokalyptiker und Integrierte. Zur kritischen Kritik der Massenkultur, Frankfurt a.M. 1986, 15-44.

${ }^{15}$ Ebd., 48.

${ }^{16}$ Ebd., 18.

17 Ebd., 34.
} 
ich - mir selbst und den anderen - beweisen, dass man über sie auch in rakademischer Manier schreiben konnte. So habe ich wohl bei den >Bildungszitaten Formulierung übertrieben (ich ging sogar so weit, dem Text über Steve Canyon ein lateinisches Zitat von Roger Bacon voranzustellen). [...] Als das Buch erschien, meinte ein italienischer Rezensent (ein äusserst gebildeter Humanist), ich hätte ungebührlich sfeine` Mittel zur Untersuchung frivoler Gegenstände benutzt. Als ob man bei der Erforschung von Affen wie Affen gestikulieren und nicht wie Darwin sprechen sollte .... ${ }^{18}$

Eco begründet sein damaliges `Übertreiben` mit der Zeit, in der das Buch entstand.

In der ebenfalls 1984 erschienenen Nachschrift zum >Namen der Roser kommt Eco nochmals auf die Scham des Lesers zu sprechen, und wie er diese als Autor umgeht:

"Du glaubst, du willst Sex und Crime und viel Action, eine spannende Krimistory, bei der am Ende herauskommt, wer der Schuldige ist, aber du würdest dich schämen, einen ehrwürdigen Schauerschinken mit schwarzen Händen des Todes und finsteren Ränkeschmieden im Klostergemäuer zu akzeptieren. Na schön, ich gebe dir einen Haufen Latein und wenig Frauen und Theologie in Hülle und Fülle und Blut in Strömen [...].«19

Auch noch im 21. Jahrhundert greift Eco auf die Wirkung des Lateinischen zurück. Auf den letzten Seiten seines illustrierten Romans Die geheimnisvolle Flamme der Königin Loana (2004), der Darstellung einer Revue populärer Figuren auf der Treppe eines Gymnasiums, lässt er den Schatten des Geistlichen Don Renato mit dem des für seine Pädagogik der Vorsorge bekannten Heiligen Don Bosco verschmelzen. Don Renato intoniert hinter dessen Rücken, "mystisch und grosszügig in seinen Ansichten, duae umbrae nobis una facta sunt, infra laternam stabimus, olim Lili Marleen, olim Lili Marleen« (490).

Eco nimmt mit diesem Roman Stellung zur Bedeutung des Populären in der Kultur. ${ }^{20}$ Wenn er dabei seinen Ich-Erzähler seitenlang über literarische Qualität parlieren und sich (als Resultat von Selbstdisziplinierung?) von offenbar allzu trivialen (und deshalb peinlichen?) Vorlieben seiner Kindheit distanzieren lässt, tritt dessen

${ }^{18}$ Ebd., 9f.

${ }^{19}$ Umberto Eco, Nachschrift zum `Namen der Rose`, München 1986, 59f.

${ }^{20}$ Vgl. Ingrid Tomkowiak: Lili Marleen auf Latein. Umberto Eco und das Populäre, in: Wo ist Kultur? Perspektiven der Kulturanalyse, hg. v. Thomas Forrer/Angelika Linke, Zürich 2014, 127-145. 
Wissen um die `kulturelle Unwürdigkeit seines Lesestoffs offen zutage.

Auf der Mailänder Buch-Vernissage am 28. Juni 2004 liest Eco aus seinem Roman, die Abbildungen flimmern über eine grosse Leinwand hinter ihm, er rezitiert, tanzt und singt die Schlager an, von denen er spricht. Den Höhepunkt des Abends ${ }^{21}$ bildet jedoch genau dieser Moment - Lili Marleen auf Latein, von Eco selbst gesungen. Denn hier wird EcosVerhältnis zum Populären überdeutlich: Er weiss um dessen Präsenz und Bedeutung, hegt auch eine gewisse Liebe zur populären Kultur, markiert jedoch akademische Distanz. Distinktion.

— Ingrid Tomkowiak ist Extraordinaria für Populäre Literaturen und Medien mit dem Schwerpunkt Kinder- und Jugendmedien am Institut für Sozialanthropologie und Empirische Kulturwissenschaft (ISEK), Abt. Populäre Kulturen, an der Universität Zürich.

${ }^{21}$ Vgl. Henning Klüver/Sigrid Löffler, Apocalypso aus Sprache und Zeichen, in: Literaturen, September 2004, online: http://www.cicero.de/salon/apocalypso-aussprache-und-zeichen/45690?seite=1 (19.07.2015). 